\title{
Morphology of synchrotron emission in young supernova remnants ${ }^{\star}$
}

\author{
G. Cassam-Chenaï ${ }^{1,2}$, A. Decourchelle ${ }^{1}$, J. Ballet $^{1}$, and D. C. Ellison ${ }^{2}$
}

\author{
${ }^{1}$ Service d'Astrophysique, CEA Saclay, 91191 Gif-sur-Yvette, France \\ 2 Department of Physics, North Carolina State University, Box 8202, Raleigh, NC 27695, USA \\ e-mail: gcassam@ncsu.edu
}

Received 9 February 2005 / Accepted 27 June 2005

\section{ABSTRACT}

In the framework of test-particle and cosmic-ray modified hydrodynamics, we calculate synchrotron emission radial profiles in young ejectadominated supernova remnants (SNRs) evolving in an ambient medium which is uniform in density and magnetic field. We find that, even without any magnetic field amplification by Raleigh-Taylor instabilities, the radio synchrotron emission peaks at the contact discontinuity because the magnetic field is compressed and is larger there than at the forward shock. The X-ray synchrotron emission sharply drops behind the forward shock as the highest energy electrons suffer severe radiative losses.

Key words. acceleration of particles - ISM: supernova remnants - ISM: cosmic rays - X-rays: ISM

\section{Introduction}

Shocks in supernova remnants (SNRs) are believed to produce the majority of the Galactic cosmic-rays (CRs) at least up to the "knee" $\left(\sim 3 \times 10^{15} \mathrm{eV}\right)$. The particle acceleration mechanism most likely responsible for this is known as diffusive shock acceleration (DSA) (e.g., Drury 1983; Blandford \& Eichler 1987). This mechanism may transfer a large fraction of the ram kinetic energy (up to 50\%) into relativistic particles and remove it from the thermal plasma (see, for example, Jones \& Ellison 1991).

Convincing observational support for the acceleration of particles in shell-type SNRs comes from their nonthermal radio and X-ray emissions due to synchrotron radiation from relativistic $\mathrm{GeV}$ and at least $\mathrm{TeV}$ electrons, respectively. In radio and X-rays, synchrotron-dominated SNRs display various morphologies: for instance, the synchrotron emission dominates in two bright limbs in SN 1006 (e.g., Rothenflug et al. 2004) whereas it is distorted and complex in RX J1713.7-3946 (e.g., Cassam-Chenaï et al. 2004b). The detection and imaging with the HESS telescopes of TeV $\gamma$-rays in RX J1713.7-3946 provides unambiguous evidence for particle acceleration to very high energies. The $\gamma$-ray morphology in this remnant is similar to that seen in X-rays (Aharonian et al. 2004).

Recent works based on Chandra (Vink \& Laming 2003, for Cas A) and XMM-Newton (Cassam-Chenaï et al. 2004a, for Kepler's SNR) observations have demonstrated that X-ray synchrotron emission is also present in ejecta-dominated SNRs

* Appendix A is only available in electronic form at http://www. edpsciences.org and largely contributes to the continuum emission at the forward shock. This X-ray emission arises from sharp filaments encircling the SNR's outer boundary. The observed width of these filaments is a few arcseconds, and has been used to constrain the magnetic field intensity just behind the shock ${ }^{1}$ (Vink \& Laming 2003; Berezhko et al. 2003; Berezhko \& Völk 2004; Völk et al. 2005; Ballet 2005).

A number of recent hydrodynamical models, including particle acceleration and photon emission, have been presented to explain various features of these observations. Reynolds (1998) has described the morphology and spectrum of the synchrotron X-ray emission from SNRs in the Sedov evolutionary phase. Similar work based on numerical simulations was done by van der Swaluw \& Achterberg (2004) who take into account the diffusion of particles. CRs are treated as test-particles in these studies.

Here, we expand on the work of Reynolds (1998) by considering young (ejecta-dominated) SNRs. We investigate the synchrotron emission morphology, both in radio and X-rays, as well as how it can be modified by efficient particle acceleration. Our results show that the radio and X-ray profiles are very different due to the effects of the magnetic field evolution and synchrotron losses in the interaction region between the contact discontinuity and the forward shock. For typical parameters, the radio emission peaks at the contact discontinuity while

${ }^{1}$ Magnetic field values are found to be at least 30 times higher than the typical Galactic field of $3 \mu \mathrm{G}$ and imply that the field has been amplified, perhaps by the particle acceleration process (Bell \& Lucek 2001). 
the X-ray emission forms sheet-like structures at the forward shock.

\section{Hydrodynamics and particle acceleration}

The hydrodynamic evolution of young supernova remnants, including the backreaction from accelerated particles, can be described by self-similar solutions if the initial density profiles in the ejected material (ejecta) and in the ambient medium have power-law distributions (Chevalier 1982, 1983), and if the acceleration efficiency (i.e. the fraction of total ram kinetic energy going into suprathermal particles) is independent of time.

Here, we use the self-similar model of Chevalier (1983) which considers a thermal gas $(\gamma=5 / 3)$ and the cosmicray fluid $(\gamma=4 / 3)$, with the boundary conditions calculated from the non-linear diffusive shock acceleration (DSA) model of Berezhko \& Ellison (1999) and Ellison et al. (2000) as described in Decourchelle et al. (2000). This acceleration model is an approximate, semi-analytical model that determines the shock modification and particle spectrum from thermal to relativistic energies in the plane-wave, steady state approximation as a function of an arbitrary injection parameter, $\eta_{\text {inj }}$ (i.e. the fraction of total particles which end up with suprathermal energies). The validity of the self-similar solutions has been discussed by Decourchelle et al. (2000) and direct comparisons between this self-similar model and the more general CR-hydro model of Ellison et al. (2004) showed good correspondence for a range of input conditions.

The hydrodynamic evolution provides the shock characteristics necessary to calculate the particle spectrum at the forward shock $^{2}$, at any time. Once a particle spectrum has been produced at the shock, it will evolve downstream because of radiative and adiabatic expansion losses. We assume that the accelerated particles remain confined to the fluid element in which they were produced, so adiabatic losses are determined directly from the fluid element expansion. The basic power law spectrum produced by DSA, before losses are taken into account, is modified at the highest energies with a exponential cutoff, $\exp \left(-p / p_{\max }\right)$, where $p_{\max }$ is determined by matching either the acceleration time to the shock age or the upstream diffusive length to some fraction of the shock radius. In our simulation, the electron-to-proton density ratio at relativistic energies, $(e / p)_{\text {rel }}$, is set equal to 0.01 (see Ellison et al. 2000).

Unless explicitly stated, our numerical examples are given for the following supernova parameters: $M_{\mathrm{ej}}=5 M_{\odot}$ for the ejected mass, $E_{51}=1$ where $E_{51}$ is the kinetic energy of the ejecta in units of $10^{51} \mathrm{erg}$ and $n=9$, where $n$ is the index of the initial power-law density profile in the ejecta $\left(\rho \propto r^{-n}\right)$. In our simulations, the SNR age is $t_{f}=400$ years and the shock velocity at the forward shock is $v_{s} \simeq 5 \times 10^{3} \mathrm{~km} \mathrm{~s}^{-1}$. For the ambient medium parameters, we take a magnetic field $B_{0}=10 \mu \mathrm{G}$, a density $n_{0}=0.1 \mathrm{~cm}^{-3}$, an ambient gas pressure $p_{\mathrm{g}, 0} / k=2300 \mathrm{~K} \mathrm{~cm}^{-3}$ and $s=0$, where $s$ is the index of its

\footnotetext{
${ }^{2}$ We do not consider CR production at the reverse shock since the magnetic field at the reverse shock may be considerably smaller than that at the forward shock due to the dilution by expansion and flux freezing of the progenitor magnetic field (see Ellison et al. 2005).
}

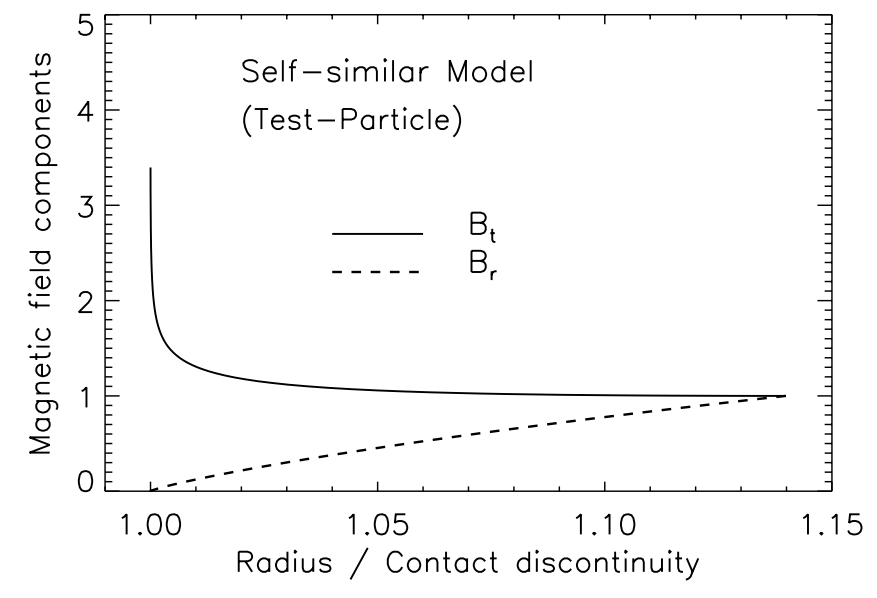

Fig. 1. Radial profile of the normal $\left(B_{\mathrm{r}}\right)$ and tangential $\left(B_{\mathrm{t}}\right)$ components of the magnetic field in a test-particle self-similar model. Each component is normalized to the forward shock.

initial power-law density profile $\left(\rho \propto r^{-s}\right)$. The case $s=0$ corresponds to a uniform interstellar medium $(s=2$ describes a stellar wind).

In the next section, we discuss the importance of the magnetic field for the synchrotron emission and particle acceleration. We do not, however, explicitly include the dynamical influence of the magnetic field on the hydrodynamics.

\section{Results}

\subsection{Magnetic field}

To track the synchrotron losses, we are interested in the temporal evolution of the magnetic field behind the shock. We assume the magnetic field to be simply compressed at the shock and passively carried by the flow, frozen in the plasma, so that it evolves conserving flux. In this simple 1-D approach, we do not consider any production of the SNR magnetic field, for instance, by hydrodynamical instabilities which is an additional effect. As for the magnetic field ahead of the forward shock, it is assumed to be isotropic and fully turbulent. Appendix A (see the on-line version) shows how to compute the magnetic field profile for self-similar solutions in both test-particle and nonlinear particle acceleration cases.

\subsubsection{Test-particle limit}

We first discuss the behavior of the normal and tangential components of the magnetic field in the test-particle case where the backreaction of the accelerated particles is neglected.

When the SNR evolves in an ambient medium which is uniform in density and magnetic field, the expansion and flux freezing generally cause the tangential component of the magnetic field to increase at the contact discontinuity whereas the normal component falls to zero (Fig. 1). As a result, the magnetic field profile is dominated by the tangential component.

One has often invoked hydrodynamic instabilities to explain the magnetic field increase at the interface between the shocked ejecta and the shocked ambient medium (Jun et al. 1995). The numerical simulations of Jun \& Norman (1996) 


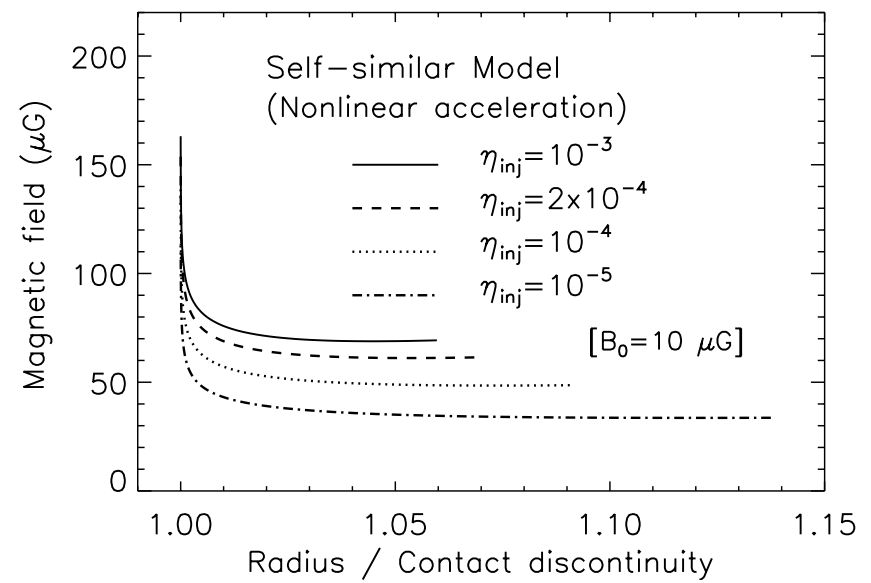

Fig. 2. Magnetic field radial profile for different values of the injection efficiency, $\eta_{\text {inj }}$, when the hydrodynamics is coupled with the non-linear DSA model. The width of the shocked region is smaller and smaller as the feedback of the accelerated particles on the SNR dynamics increases.

have shown that the magnetic field could be amplified by a factor 60 by Rayleigh-Taylor and Kelvin-Helmholtz instabilities. Here, we note that simple advection of the magnetic field already predicts amplification by a factor 5 (Table A.1 top, $n=9$ ).

We note that, if the SNR evolves in a wind with a decreasing initial density profile, advection goes the other way (diluting the magnetic field instead of amplifying it). But when both the ambient density and magnetic field decrease with radius, as would be the case for a pre-supernova stellar wind, the magnetic field is larger close to the contact discontinuity than at the forward shock (by a factor of $\sim 1000$ in some cases). This is because the dilution of the advected magnetic field is negligible compared to the fact that the ambient magnetic field was much larger at early times.

\subsubsection{Nonlinear particle acceleration}

We now consider the behavior of the normal and tangential components of the magnetic field in the nonlinear case where the backreaction of the accelerated particles on the shock is taken into account.

In the ideal non-linear case, where the acceleration is instantaneous, the magnetic field diverges at the contact discontinuity because of its tangential component, whatever the injection efficiency is, as in the test-particle case. However, the contrast between the magnetic field in a given fluid element and the one just behind the shock, will be always smaller than in the test-particle case (see Table A.1). Figure 2 shows the profile of the total downstream magnetic field for different values of the injection efficiency. Table 1 shows the associated compression ratio and immediate post-shock magnetic field.

\subsection{Synchrotron emission}

Once the magnetic field structure and the particle spectrum (attached to a fluid element) modified by the radiative and
Table 1. Compression ratio, $r_{\text {tot }}$, and downstream magnetic field, $B_{s}$, at the forward shock obtained for different injection, $\eta_{\text {inj. }}$. The magnetic field compression ratio is given by $r_{B} \equiv B_{s} / B_{0}=\sqrt{1 / 3+2 r_{\text {tot }}^{2} / 3}$ and $B_{0}=10 \mu \mathrm{G}$ here.

\begin{tabular}{lcccc}
\hline \hline$\eta_{\text {inj }}$ & $10^{-3}$ & $2 \times 10^{-4}$ & $10^{-4}$ & $10^{-5}$ \\
\hline$r_{\text {tot }}$ & 8.5 & 7.5 & 5.9 & 4.1 \\
$B_{s}(\mu \mathrm{G})$ & 69 & 61 & 49 & 34 \\
\hline
\end{tabular}
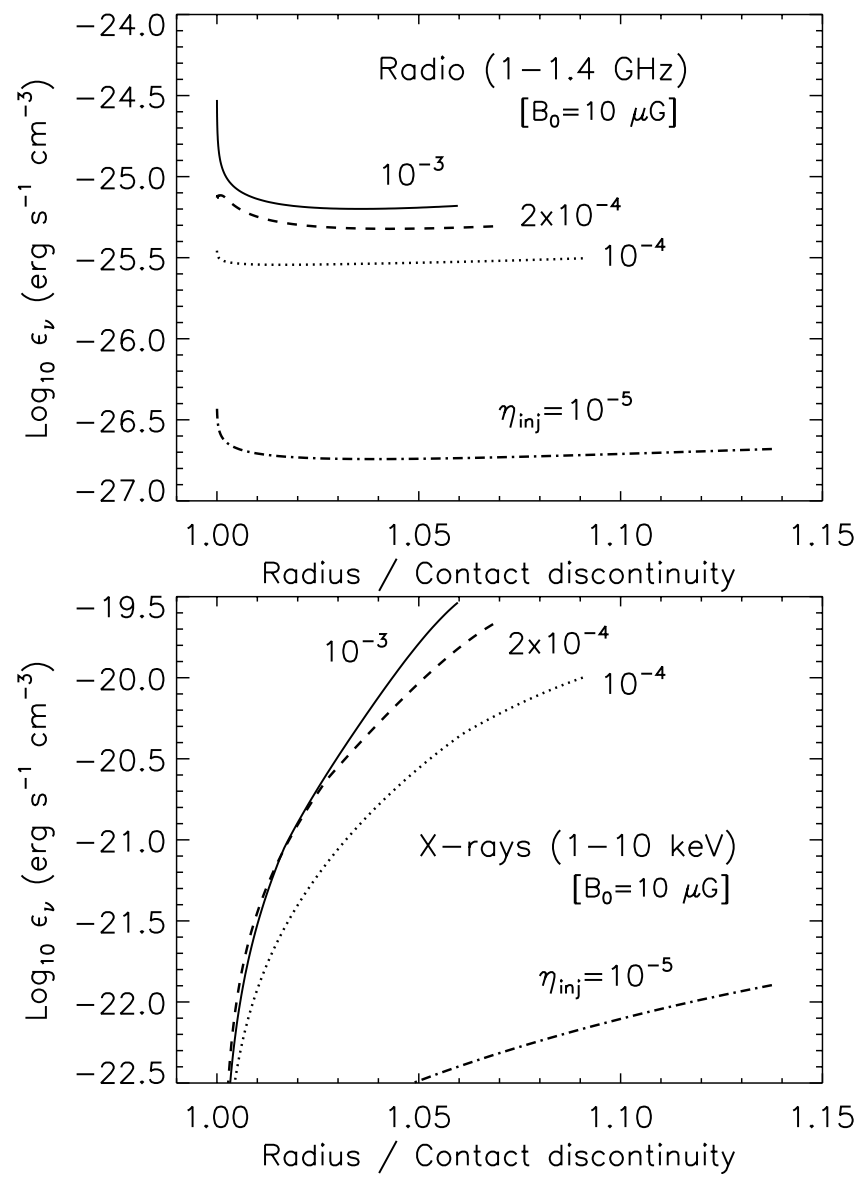

Fig. 3. Radio (top panel) and X-ray (bottom panel) synchrotron volume emissivity, $\epsilon_{v}$, radial profile for different injection efficiencies.

adiabatic expansion losses as computed in Reynolds (1998) are known, we compute the synchrotron emission (Rybicki \& Lightman 1979), averaged over the pitch-angle, in any energy band $^{3}$.

Figure 3 shows the radial profiles of the synchrotron emission in the radio (top panel) and X-ray (bottom panel) domains for different injection efficiencies, $\eta_{\text {inj. }}$. An increase in the injection efficiency not only provides a larger number of accelerated electrons, but also a larger compression of the downstream magnetic field (see Table 1) and a narrower interaction region. These effects combine to produce enhanced synchrotron emission as the injection increases.

The radio synchrotron emission is produced by $\mathrm{GeV}$ electrons which are not affected by radiative losses. Consequently, the radio synchrotron emission critically depends on the final

${ }^{3}$ We did not calculate the synchrotron emission from the precursor. 


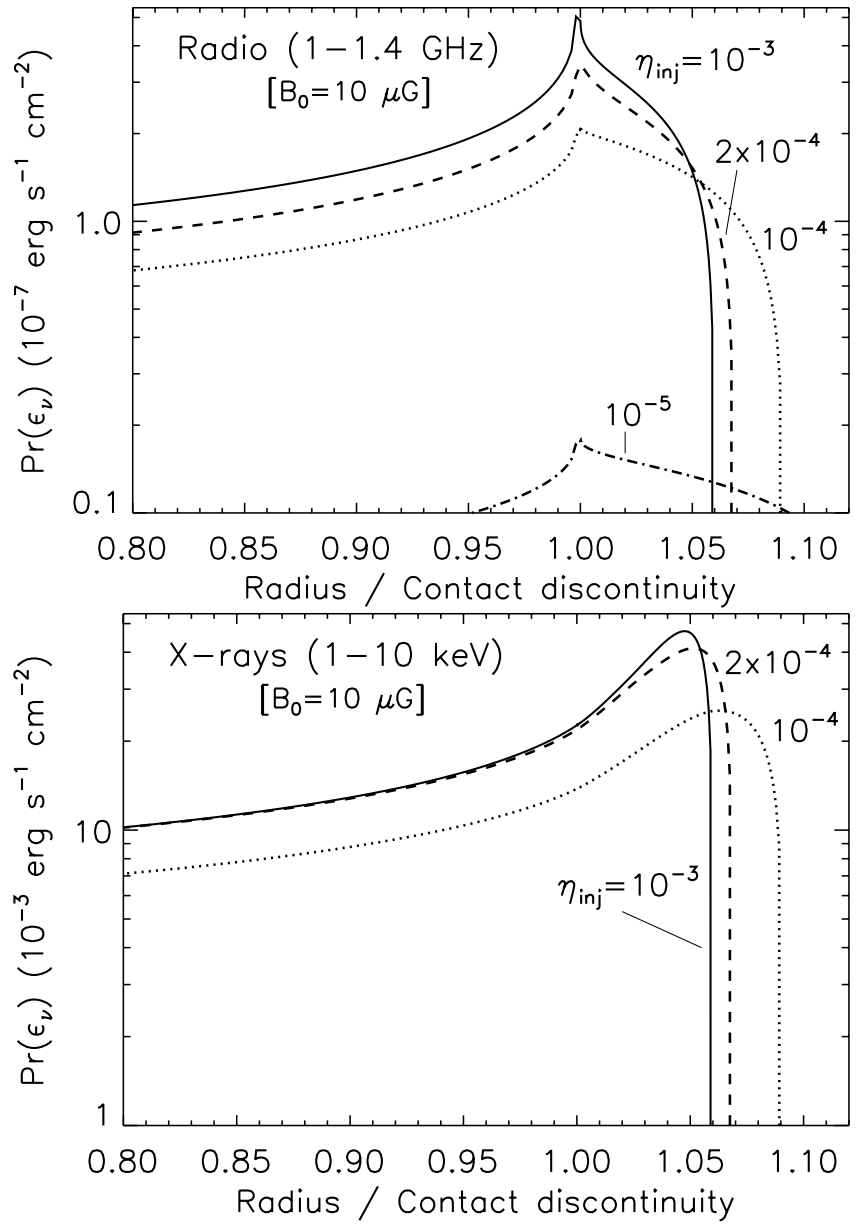

Fig. 4. Radio (top panel) and X-ray (bottom panel) synchrotron volume emissivity radial profile after projection onto the line-of-sight for different injection efficiencies. Emission from particles accelerated at the reverse shock is assumed to be negligible.

magnetic field profile (Fig. 2) and, therefore, peaks at the contact discontinuity. In contrast, the X-ray synchrotron emission is produced by the highest momentum electrons $\left(\sim 10^{3-5} \mathrm{~m}_{\mathrm{p}} c\right)$ which, depending on the downstream field strength, may suffer radiative losses. The high energy electrons that have been accelerated at the earliest time have suffered strong synchrotron losses as they were advected behind the shock. Because of this, they are not numerous enough at the end to radiate in the X-ray regime despite a strong magnetic field. As a result, the X-ray synchrotron emission rapidly decreases behind the shock. The $\mathrm{X}$-ray profile becomes sharper when the injection efficiency increases because it provides larger compression of the downstream magnetic field and then stronger synchrotron losses.

Figure 4 shows the synchrotron emission after integration along the line-of-sight. The radial profile of the radio emission (top panel) shows a peak at the contact discontinuity. The radial profile of the X-ray projected synchrotron emission (bottom panel) shows bright rims just behind the forward shock whose width decreases as the injection efficiency increases.

\section{Discussion and conclusion}

We have computed the radio and X-ray synchrotron emission in young ejecta-dominated SNRs. This has been done using a one dimensional, self-similar hydrodynamical calculation coupled with a non-linear diffusive shock acceleration model, and taking into account the adiabatic and radiative losses of the electron spectrum during its advection in the remnant.

We show that the morphology of the synchrotron emission in young ejecta-dominated SNRs is very different in radio and $\mathrm{X}$-ray. This is the result of the increased magnetic field toward the contact discontinuity, to which only low energy electrons that emit radio are sensitive, while the high energy electrons emitting $\mathrm{X}$-rays experience strong radiative losses and are mostly dependent on the post-shock magnetic field.

Briefly, the radio synchrotron emission increases as one moves from the forward shock toward the contact discontinuity due to a compression of the magnetic field (particularly its tangential component), assuming both uniform ambient density and upstream magnetic field. Such a compression naturally results from the dynamical evolution of the SNR. In contrast, because of the radiative losses, the $\mathrm{X}$-ray synchrotron emission decreases behind the forward shock and forms sheet-like structures after line-of-sight projection. Their widths decrease as the acceleration becomes more efficient.

The morphology of the radio synchrotron emission obtained for the young ejecta-dominated stage of SNRs will differ from that of SNRs in the Sedov phase (but not in X-ray). Indeed, Reynolds (1998) has shown that both the normal and tangential components of the magnetic field decrease behind the forward shock in the Sedov phase and, as a result, we expect the radio synchrotron emission to decrease behind the shock (however, less rapidly than the X-ray synchrotron emission since the radio electrons do not experience radiative losses).

Our model qualitatively reproduces the main features of the radio and X-ray observations of emission in young ejectadominated SNRs (e.g., Tycho \& Kepler), i.e. bright radio synchrotron emission at the interface between the shocked ejecta and ambient medium, and a narrow filament of X-ray emission at the forward shock. However, this model is unable to reproduce the thin radio filaments observed at the forward shock in some SNRs (for instance those seen in Tycho's SNR, Dickel et al. 1991).

We note that extensions of this work to cases with exponential ejecta profiles and/or SNRs evolving in a pre-supernova stellar wind with varying magnetic fields, cannot be done with self-similar solutions. These cases can be calculated in the numerical CR-modified hydrodynamical model described in Ellison et al. (2005) and this work is in progress (Ellison \& Cassam-Chenaï 2005).

\section{References}

Aharonian, F. A., Akhperjanian, A. G., Aye, K.-M., et al. 2004, Nature, 432, 75

Ballet, J. 2005, Space Sci. Rev., accepted,

[arXiv: astro-ph/0503309]

Bell, A. R., \& Lucek, S. G. 2001, MNRAS, 321, 433 
Berezhko, E. G., \& Ellison, D. C. 1999, ApJ, 526, 385

Berezhko, E. G., Ksenofontov, L. T., \& Völk, H. J. 2002, A\&A, 395, 943

Berezhko, E. G., Ksenofontov, L. T., \& Völk, H. J. 2003, A\&A, 412, L11

Berezhko, E. G. \& Völk, H. J. 2004, A\&A, 419, L27

Blandford, R. D. \& Eichler, D. 1987, Phys. Rep., 154, 1

Cassam-Chenaï, G., Decourchelle, A., Ballet, J., et al. 2004a, A\&A, 414,545

Cassam-Chenaï, G., Decourchelle, A., Ballet, J., et al. 2004b, A\&A, 427, 199

Chevalier, R. A. 1982, ApJ, 258, 790

Chevalier, R. A. 1983, ApJ, 272, 765

Decourchelle, A., Ellison, D. C., \& Ballet, J. 2000, ApJ, 543, L57

Dickel, J. R., van Breugel, W. J. M., \& Strom, R. G. 1991, AJ, 101, 2151

Drury, L. O. 1983, Rep. Prog. Phys., 46, 973
Ellison, D. C., Berezhko, E. G., \& Baring, M. G. 2000, ApJ, 540, 292

Ellison, D. C., \& Cassam-Chenaï, G. 2005, ApJ, accepted

Ellison, D. C., Decourchelle, A., \& Ballet, J. 2004, A\&A, 413, 189

Ellison, D. C., Decourchelle, A., \& Ballet, J. 2005, A\&A, 429, 569

Jones, F. C., \& Ellison, D. C. 1991, Space Sci. Rev., 58, 259

Jun, B.-I., \& Norman, M. L. 1996, ApJ, 465, 800

Jun, B.-I., Norman, M. L., \& Stone, J. M. 1995, ApJ, 453, 332

Lyutikov, M. \& Pohl, M. 2004, ApJ, 609, 785

Reynolds, S. P. 1998, ApJ, 493, 375

Reynolds, S. P., \& Chevalier, R. A. 1981, ApJ, 245, 912

Rothenflug, R., Ballet, J., Dubner, G., et al. 2004, A\&A, 425, 121

Rybicki, G. B., \& Lightman, A. P. 1979, Radiative processes in astrophysics (New York: Wiley-Interscience)

Völk, H. J., Berezhko, E. G., \& Ksenofontov, L. T. 2005, A\&A, 433, 229

van der Swaluw, E., \& Achterberg, A. 2004, A\&A, 421, 1021

Vink, J., \& Laming, J. M. 2003, ApJ, 584, 758 
G. Cassam-Chenaï et al.: Morphology of synchrotron emission in young SNRs, Online Material p 1

\section{Online Material}




\section{Appendix A: Magnetic field evolution}

The evolution of the normal (subscript $r$ ) and tangential (subscript t) components of the magnetic field at the downstream position, $B$, is given by (Reynolds \& Chevalier 1981):

$$
\begin{aligned}
& B_{\mathrm{r}}(r)=B_{\mathrm{r}, j}\left(\frac{r}{r_{j}}\right)^{-2} \\
& B_{\mathrm{t}}(r)=B_{\mathrm{t}, j} \frac{\rho}{\rho_{j}} \frac{r}{r_{j}}
\end{aligned}
$$

and the total magnetic field is simply (Reynolds 1998):

$$
B(r)=\left(B_{\mathrm{r}}(r)^{2}+B_{\mathrm{t}}(r)^{2}\right)^{1 / 2}
$$

In these equations, $r$ and $\rho$ are, respectively, the radius and density of a fluid element at the current time that was shocked at the previous time $t_{j}$. At time $t_{j}$, the fluid element was just behind the shock at the radius $r_{j}$, with a density $\rho_{j}$ and a magnetic field $B_{j}$.

We assume that the upstream magnetic field at time $t_{j}, B_{0, j}$ is isotropic and fully turbulent so that the components of the immediate post-shock magnetic field $B_{j}$ in Eqs. (A.1) and (A.2) are given on average by (Berezhko et al. 2002):

$$
\begin{aligned}
& B_{\mathrm{r}, j}=1 / \sqrt{3} B_{0, j} \\
& B_{\mathrm{t}, j}=\sqrt{2 / 3} r_{\mathrm{tot}} B_{0, j},
\end{aligned}
$$

where $r_{\text {tot }}$ is the shock compression ratio. In the self-similar approach, $r_{\text {tot }}$ is assumed independent of time (see Decourchelle et al. 2000, for details).

We consider that the current magnetic field upstream of the forward shock, $B_{0, s}$, can behave like:

$B_{0, s}=B_{0, j}\left(\frac{r_{s}}{r_{j}}\right)^{-q}$

where $r_{s}$ is the current shock radius. If the magnetic field is uniform, the index $q$ is equal to 0 . In a stellar wind $(s=2)$, the magnetic field profile may be decreasing yielding $q=1$ (Lyutikov \& Pohl 2004) or $q=2$ if we assume that it is frozen in the plasma.

We define the magnetic field contrast factor, $\sigma_{B} \equiv B / B_{S}$, as the ratio between the current magnetic field in a fluid element, $B$, and the current one just behind the shock, $B_{s}$. We have:

$\sigma_{B}=\left(\frac{\sigma_{B_{\mathrm{r}}}^{2}+2 r_{\mathrm{tot}}^{2} \sigma_{B_{\mathrm{t}}}^{2}}{1+2 r_{\mathrm{tot}}^{2}}\right)^{1 / 2}$

where $\sigma_{B_{\mathrm{r}}} \equiv B_{\mathrm{r}} / B_{\mathrm{r}, s}$ and $\sigma_{B_{\mathrm{t}}} \equiv B_{\mathrm{t}} / B_{\mathrm{t}, s}$ are the magnetic field contrast factors of the normal and tangential components of the field, respectively. The components $B_{\mathrm{r}, s}$ and $B_{\mathrm{t}, s}$ obey the same relation as in Eqs. (A.4) and (A.5).

\section{A.1. Test-particle limit}

Assuming adiabaticity of the thermal gas, the magnetic field contrast factors of the normal and tangential components of the field are given by:

$$
\begin{aligned}
\sigma_{B_{\mathrm{r}}} & =\left(\frac{R_{s}}{R}\right)^{2}\left(\frac{v_{j}}{v_{s}}\right)^{\beta_{\mathrm{r}}} \\
\sigma_{B_{\mathrm{t}}} & =\left(\frac{P_{\mathrm{g}, s}}{P_{\mathrm{g}}}\right)^{-3 / 5}\left(\frac{R_{s}}{R}\right)^{-(11-3 s) / 5}\left(\frac{v_{j}}{v_{s}}\right)^{\beta_{\mathrm{t}}}
\end{aligned}
$$

where the indexes $\beta_{\mathrm{r}}$ and $\beta_{\mathrm{t}}$ are given by:

$$
\begin{aligned}
& \beta_{\mathrm{r}}=(q-2) \frac{n-3}{3-s} \\
& \beta_{\mathrm{t}}=\frac{5 n-33-3 s(n-5)}{5(3-s)}+q \frac{n-3}{3-s} .
\end{aligned}
$$

In Eqs. (A.8) and (A.9), $R_{s} / R$ and $P_{\mathrm{g}, s} / P_{\mathrm{g}}$ are the ratio of the self-similar radii and thermal gas pressures, respectively, between the shock (subscript $s$ ) and a fluid element (see Chevalier 1982). They depend on $n$ and $s$, but also weakly on $v_{j} / v_{s}$ where $v_{s}$ and $v_{j}$ are the current shock velocity and the shock velocity at the time $t_{j}$, respectively.

In the framework of these self-similar solutions, the forward shock velocity tends to infinity at early times, corresponding to fluid elements close to the contact discontinuity at the current time. To limit the maximum velocity to a realistic value, we look at the value of $\sigma_{B}$ for a shock velocity ratio $v_{j} / v_{s}=10$. For the typical forward shock velocity $v_{s}$ that we have used for the numerical application, the initial velocity corresponds to $v_{j} \simeq 5 \times 10^{4} \mathrm{~km} \mathrm{~s}^{-1}$. This shock velocity is the criterion used to define the radial position of the oldest fluid element that is currently located close to the contact discontinuity.

Here, we consider the case of both an uniform ambient medium $(s=0)$ and upstream magnetic field $(q=0)$. Under this assumption, $B_{s}=B_{j}$, since $r_{\text {tot }}$ is constant with time. Then, the magnetic field contrast factor, $\sigma_{B}$ is equal to $B / B_{j}$ and can be viewed as a compression or a dilution factor. Table A.1 (top) gives the contrast $\sigma_{B}$ for different values of $n$.

\section{A.2. Nonlinear particle acceleration}

In the ideal non-linear case, where the acceleration is instantaneous and efficient, the thermal gas pressure falls to zero at the contact discontinuity while the relativistic gas pressure goes to infinity. Hence, the contrast factor of the tangential field component, $\sigma_{B_{\mathrm{t}}}$, given by Eq. (A.9), obtained in the test-particle limit, is not defined when $v_{j} / v_{s}$ tends to infinity.

However, the contrast of the tangential component of the magnetic field can also be found by using the adiabaticity of the relativistic gas:

$\sigma_{B_{\mathrm{t}}}=\left(\frac{P_{\mathrm{c}, s}}{P_{\mathrm{c}}}\right)^{-3 / 4}\left(\frac{R_{s}}{R}\right)^{-(10-3 s) / 4}\left(\frac{v_{j}}{v_{s}}\right)^{\beta_{\mathrm{t}}^{\prime}}$

where the index $\beta_{t}^{\prime}$ is given by:

$\beta_{\mathrm{t}}^{\prime}=\frac{4 n-30-3 s(n-5)}{4(3-s)}+q \frac{n-3}{3-s}$.

In Eq. (A.12), $P_{\mathrm{c}, s} / P_{\mathrm{c}}$ is the ratio of the self-similar relativistic gas pressures between the shock (subscript $s$ ) and a fluid element. This ratio depends on $n, s$, and $v_{j} / v_{s}$. The contrast of the 
Table A.1. Test-particle: Magnetic field contrast factor, $\sigma_{B}$, for a velocity ratio $v_{j} / v_{s}=10$, radius ratio and self-similar thermal gas pressure ratio (see Chevalier 1982) and shock velocity, for different values of the indexes $n(s=0$ and $q=0)$. Nonlinear DSA: same as Test-particle but with lower and upper limits on the magnetic field contrast factor, $\sigma_{B}$, with $\eta_{\text {inj }}=10^{-3}$ (see Sect. A. 2 for more explanations).

\begin{tabular}{lccccccc}
\hline \hline & $n$ & $R_{s} / R$ & $P_{\mathrm{g}, s} / P_{\mathrm{g}}$ & $\sigma_{B}$ & $v_{s}\left(\mathrm{~km} \mathrm{~s}^{-1}\right)$ \\
\hline \multirow{3}{*}{ Test-particle } & 7 & 1.181 & 0.964 & 0.95 & 4840 \\
& 9 & 1.140 & 0.885 & \multicolumn{2}{c}{5.0} & 4850 \\
& 12 & 1.121 & \multicolumn{2}{c}{0.836} & \multicolumn{2}{c}{54} & 4940 \\
\hline \multirow{3}{*}{ Nonlinear DSA } & $n$ & $R_{s} / R$ & $P_{s} / P$ & $P_{\mathrm{c}, s} / P$ & $\min \left(\sigma_{B}\right)$ & $\max \left(\sigma_{B}\right)$ & $v_{s}\left(\mathrm{~km} \mathrm{~s}^{-1}\right)$ \\
& 7 & 1.080 & 1.135 & 0.806 & 0.50 & 0.66 & 4370 \\
& 9 & 1.060 & 1.045 & 0.754 & 2.6 & 3.4 & 4470 \\
& 12 & 1.051 & 0.988 & 0.714 & 28 & 36 & 4610 \\
\hline
\end{tabular}

normal field component, $\sigma_{B_{r}}$, is still given by Eq. (A.8). The asymptotic behavior of the contrast factor, $\sigma_{B_{\mathrm{t}}}$, can be derived from Eq. (A.12) because the relativistic gas pressure does not tend to zero at the contact discontinuity.

Because the thermal gas pressure vanishes as we approach the contact discontinuity in the case of ideal particle acceleration, i.e., when the acceleration is instantaneous and efficient, the contrast of the tangential field component, $\sigma_{B_{\mathrm{t}}}$, will always be smaller than in the test-particle case where the thermal gas pressure rapidly tends to a constant (see Eq. A.9). Table A.1 (bottom) gives the lower and upper limits on the magnetic field contrast factor, $\sigma_{B}$, in the case of ideal nonlinear particle acceleration for $\eta_{\text {inj }}=10^{-3}$ and for different values of $n$ when both the ambient medium and upstream magnetic field are uniform ( $s=0$ and $q=0$ ). The lower and upper limits on $\sigma_{B}$ are obtained by replacing in Eq. (A.12) the ratio of the selfsimilar relativistic gas pressures, $P_{\mathrm{c}, s} / P_{\mathrm{c}}$, by the ratio of the self-similar total gas pressures, $P_{s} / P \equiv\left(P_{\mathrm{c}, s}+P_{\mathrm{g}, s}\right) /\left(P_{\mathrm{c}}+P_{\mathrm{g}}\right)$, and by the ratio between the self-similar relativistic gas pressure at the shock and the self-similar total gas pressure, $P_{c, s} / P$, respectively.

However, for an injection efficiency lower than $\sim 5 \times 10^{-4}$, the acceleration is not efficient enough for the shock to be modified at the beginning of the evolution. In that case, the fluid elements that have been shocked at the earliest times are still dominated by the thermal gas so that test-particle solutions could still apply locally. 